\title{
Abiotrophia defectiva as a Rare Causative Agent of Periprosthetic Total Knee Arthroplasty Infections: A Case Report and Literature Review
}

\author{
Emre Kocazeybek ${ }^{1} \quad$ Mehmet Demirel $^{1}$
Serap Simsek Yavuz ${ }^{2} \quad$ Mehmet Asik $^{1}$
}

Mehmet Ersin ${ }^{1}$ Omer Naci Ergin ${ }^{1} \quad$ Betul Sadic ${ }^{2}$
Address for correspondence Emre Kocazeybek, MD, Department of Orthopedics and Traumatology, Istanbul Medical Faculty, Istanbul University, Istanbul, 34098, Turkey (e-mail: emrekczb@gmail.com).

J Lab Physicians:2020;12:219-221

\author{
Abstract \\ Keywords \\ - Abiotrophia defectiva \\ - osteoarticular \\ infections \\ - MALDI-TOF MS
}

We present a case of Abiotrophia defectiva in a prosthetic knee infection following total knee replacement for the first time. A 69-year-old female was prediagnosed with prosthetic knee infection, and a two-stage revision arthroplasty was applied. A. defectiva was cultured by matrix-assisted laser desorption ionization-time of flight mass spectrometry (MALDI-TOF MS) from the synovial fluid aspirates. Penicillin $G$ and gentamicin had been administered. One year postoperatively, a scintigraphy showed no recurrence. $A$. defectiva may be missed in culture negative patients with knee or hip arthroplasty. They should be carefully evaluated if they have undergone recent dental procedures.

\section{Introduction}

Abiotrophia defectiva is a member of the human normal flora, which colonizes the oral cavity, intestinal, and genitourinary tracts. Its culture requires special conditions. ${ }^{1}$

A. defectiva specifically targets endovascular structures, and it is reported to be a key agent in cases of culture-negative endocarditis., ${ }^{2,3}$ Furthermore, this fastidious pathogen has been associated with osteoarticular and prosthetic joint infections. Knee prosthesis infections caused by A. defectiva have been reported sporadically. ${ }^{1,4}$

The purpose of this report was to describe the clinical, radiological, and microbiological features of an exceptional case of total knee arthroplasty (TKA) infection due to A. defectiva.

\section{Case History}

A 69-year-old female who underwent a TKA a year ago was admitted with the complaints of pain, erythema, and swelling in the operated knee. She had no relevant medical history except a tooth extraction that occurred 3 months before the operation.
A plain radiograph was normal. Additionally, laboratory findings included high-levels of C-reactive protein (38 $\mathrm{mg} / \mathrm{L})$, erythrocyte sedimentation rate at $58 \mathrm{~mm} / \mathrm{h}$, and normal white blood count ( 7800 cells/mL). The initial diagnosis was periprosthetic TKA infection. No bacterial growth was yielded in any of the cultivations. A two-stage revision arthroplasty was applied.

In the first stage revision, a debridement of the infected area, removal of implants, and insertion of an antibiotic-impregnated cement spacer were performed. Meanwhile, synovial fluid aspirates were sent for microbiological analysis.

\section{Laboratory Investigations}

Intraoperative synovial fluid was inoculated into aerobic (BD BACTEC Plus Aerobic/F, USA) and anaerobic (BD BACTEC Lytic/10 Anaerobic/F, USA) blood-culture bottles and incubated in automatic chambers for 14 days. The aerobic bottle produced Gram-positive cocci after a 5-day incubation at $37^{\circ} \mathrm{C}$, and highly pleomorphic coccobacilli in small chains were recognized after subculturing of the aerobic bottle in chocolate agar with supplemented pyridoxal $37^{\circ} \mathrm{C}$ under
DOI https://doi.org/ 10.1055/s-0040-1720942 ISSN 0974-2727. (c) 2020. The Indian Association of Laboratory Physicians.

This is an open access article published by Thieme under the terms of the Creative Commons Attribution-NonDerivative-NonCommercial-License, permitting copying and reproduction so long as the original work is given appropriate credit. Contents may not be used for commercial purposes, or adapted, remixed, transformed or built upon. (https://creativecommons.org/licenses/by-nc-nd/4.0/)

Thieme Medical and Scientific Publishers Pvt. Ltd., A-12, 2nd Floor, Sector 2, Noida-201301 UP, India 
a 5\% CO2 atmosphere for 24 hours. No growth was observed in $5 \%$ sheep-blood agar. The biochemical test results for the colonies were as follows: negative catalase and oxidase tests, and no growth on media with bile-esculin and $6.5 \% \mathrm{NaCl}$. Furthermore, although small colonies in small chains were observed in chocolate agar plate, these colonies were not identified by conventional identification methods or rapid identification systems including API Coryne and Rapid ID 32 Strep (bioMérieux, France).

Therefore, matrix-assisted laser desorption ionization-time of flight mass spectrometry (MALDI-TOF MS) analysis was performed with a MicroFlex LT mass spectrometer (Bruker Daltonics, Bremen, Germany). Each colony was overlaid with $1 \mu \mathrm{L}$ of matrix solution, and mass spectra of the sample was then transferred to the Biotyper software (version 3.0; Bruker, Karlsruhe, Germany). The analyses were performed by comparing with other spectra in the Bruker database library. A. defectiva was identified with a homology of $99.9 \%$. Susceptibility testing was performed on MuellerHinton agar with pyridoxal-supplemented sheep blood $\left(\mathrm{CO}_{2}\right.$ $5 \%, 37^{\circ} \mathrm{C}, 24$ hour), according to the guidelines of The Clinical and Laboratory Standards Institute (CLSI) for disk diffusion, and $A$. defectiva was detected to be susceptible to the all following antibiotics: penicillin $\mathrm{G}$, erythromycin, imipenem, cefotaxime, ceftaroline, ceftriaxone, clindamycin, daptomycin, gentamicin, levofloxacin, linezolid, meropenem, penicillin, streptomycin, and vancomycin, with the exception of tetracycline. Minimal inhibitory concentrations obtained with the E-test (bioMérieux, Durham, NC, USA) were as follows: penicillin G, $0.032 \mathrm{mg} / \mathrm{L}$; gentamicin, $4 \mathrm{mg} / \mathrm{L}$ ). A combination of gentamicin and penicillin (first-line treatment) treatment was advised by the American Heart Association (AHA) guidelines for the treatment of infective endocarditis caused by Abiotrophia species ${ }^{5}$

After identifying the pathogen, based on drug susceptibility profile of $A$. defectiva, $4 \times 1000.000 \mathrm{IU} / \mathrm{mL}$ penicillin $\mathrm{G}$ and $3 \times 80 \mathrm{mg} / \mathrm{L}$ gentamicin IV had been administered parenterally for 30 days until acute phase reactants were normal and patient's physical examination was clinically stable. Because of the increased risk for endovascular infections in patients infected with $A$. defectiva, endocarditis was ruled out through an echocardiography. At 12 months of follow-up, considering normal levels of acute-phase reactants, the second stage of the revision arthroplasty was applied using a hinged knee prosthesis. One year postoperatively, a labeled leukocyte scintigraphy showed no evidence of recurrence. With a satisfactory functional condition, the patient returned to her daily life without any limitations. The knee society and The Western Ontario and McMaster Universities Osteoarthritis Index (WOMAC) score were preop 68 and 78, respectively.

Ethical approval was not necessary for case reports in our institution, and patient consent has been obtained.

\section{Discussion}

Exopolysaccharide and fibronectin are key virulence factors of A. defectiva and provide a strong binding capacity to endovascular structures and prosthetic surface. ${ }^{3,4}$ Although there are a vast number of case reports dealing with Abiotrophia endocarditis in the medical literature, Abiotrophia infections associated with prosthetic joint replacement are uncommon and have been sporadically reported. ${ }^{4}$ To the best of our knowledge, the literature has described only a few of orthopaedic prosthesis-associated $A$. defectiva infections to date

Abiotrophia infections associated with prosthetic joint replacement are uncommon. ${ }^{4}$

A 71-year-old man with a TKA infection had a negative bacterial culture, and $A$. defectiva was identified by $16 \mathrm{~S}$ rDNA sequencing. The patient had underwent a dental intervention 3 weeks before the admission. ${ }^{6}$ Similarly, a 65-year-old woman with TKA infection due to A. defective was identified by the PCR method. ${ }^{7}$ Another example was a case of a total hip arthroplasty infection caused by A. defectiva. ${ }^{8}$ MALDI-TOF MS offers a faster alternative for the detection of $A$. defectiva. ${ }^{9}$ In an infective endocarditis case, MALDI-TOF MS presented sufficient ability to identify A. defectiva. ${ }^{10}$ Therefore, we preferred to use MALDITOF-MS for the identification of A. defectiva.

It was previously reported that dental procedures were not risk factors for subsequent total hip or knee infection. ${ }^{11}$ However, our patient had a tooth extraction 3 months before their TKA. Therefore, we may suggest that Abiotrophia infection may have originated from the oral flora, but the clinical signs started 9 months after the TKA. Cassir et $\mathrm{al}^{6}$ reported that a 71 -year-old patient with a total knee prosthesis underwent a dental treatment without antibiotic prophylaxis 3 weeks before the isolation of $A$. defectiva. The patient had infection signs before the dental procedure and concluded that the dental procedure may not be the origin. We are agree with the conclusions of Berbari et al and Cassir et al for ignoring the role of dental procedures in prosthetic hip or knee infections. ${ }^{6,11}$ A mediate or high-penicillin resistance have been reported in A. defective strains, but our strain was highly susceptible to penicillin G. ${ }^{12}$

We used MALDI-TOF MS in place of 16S rRNA-based PCR. Protocol for the diagnosis of prosthetic joint infection using an "arthritis kit" procedure was advised by Cassir et al. ${ }^{6}$ A. defectiva may be neglected in these infections because of its fastidious nature, and culture negative infections in patients with knee or hip arthroplasty should be carefully evaluated.

\section{Financial support}

This study received no financial support.

\section{Conflicts of Interest}

None declared

\section{References}

1 Gupta P, Agstam S, Angrup A, Manoj RK, Kanaujia R, Ray P. Infective endocarditis caused by Abiotrophia defectiva presenting as anterior mitral leaflet perforation mimicking cleft anterior mitral leaflet. J Family Med Prim Care 2020;9(2):1229-1231

2 Chroboczek T, Le Scanff J, David G. First case of Abiotophia defectiva infectious endocarditis treated with a combination of amoxicillin and daptomycin. J Chemother 2020 (e-pub ahead of print). doi: 10.1080/1120009X.2020.1734163 
3 Elashery AR, Stratidis J, Patel AD. Double-valve heart disease and glomerulonephritis consequent to Abiotrophia defectiva endocarditis. Tex Heart Inst J 2020;47(1):35-37

4 Tooley TR, Siljander MP, Hubers M. Development of a periprosthetic joint infection by Abiotrophia defectiva years after total knee arthroplasty. Arthroplast Today 2019;5(1):49-51

5 Baddour LM, Wilson WR, Bayer AS, et al; American Heart Association Committee on Rheumatic Fever, Endocarditis, and Kawasaki Disease of the Council on Cardiovascular Disease in the Young, Council on Clinical Cardiology, Council on Cardiovascular Surgery and Anesthesia, and Stroke Council. Infective endocarditis in adults: diagnosis, antimicrobial therapy, and management of complications: A Scientific Statement for Healthcare Professionals from the American Heart Association. Circulation 2015;132(15):1435-1486

6 Cassir N, Grillo JC, Argenson JN, Drancourt M, Levy PY. Abiotrophia defectiva knee prosthesis infection: a case report. J Med Case Reports 2011;5:438

7 Ince A, Tiemer B, Gille J, Boos C, Russlies M. Total knee arthroplasty infection due to Abiotrophia defectiva. J Med Microbiol 2002;51(10):899-902
8 Rozemeijer W, Jiya TU, Rijnsburger M, Heddema E, Savelkoul P, Ang W. Abiotrophia defectiva infection of a total hip arthroplasty diagnosed by 16S rRNA gene sequencing. Diagn Microbiol Infect Dis 2011;70(1):142-144

9 Giannakopoulos K, Zompolou C, Behnes M, Elmas E, Borggrefe M, Akin I. Infective endocarditis - A word of caution on non-typical bacteria. Eur Rev Med Pharmacol Sci 2016;20(22):4782-4785

10 Holler JG, Pedersen LK, Calum H, et al. Using MALDI-TOF mass spectrometry as a rapid and accurate diagnostic tool in infective endocarditis: a case report of a patient with mitral valve infective endocarditis caused by Abiotrophia defectiva. Scand J Infect Dis 2011;43(3):234-237

11 Berbari EF, Osmon DR, Carr A, et al. Dental procedures as risk factors for prosthetic hip or knee infection: a hospital-based prospective case-control study. Clin Infect Dis 2010;50(1):8-16

12 Rudrappa M, Kokatnur L. Infective endocarditis due to Abiotrophia defectiva and its feared complications in an immunocompetent person: rare, but real. J Glob Infect Dis $2017 ; 9(2): 79-81$ 